\section{Les isoformes de PML et la réponse au TGF- $\beta$}

Faten $\varepsilon l-A s m i$, Mounira K. Chelbi-Alix

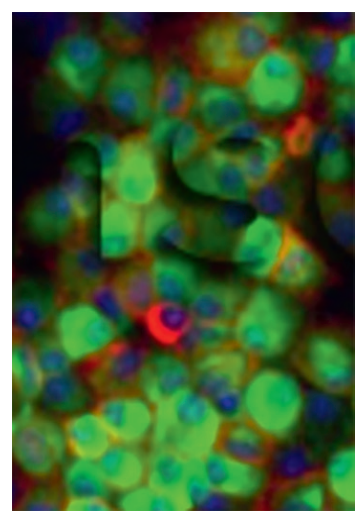

Inserm UMR-S 1124, Université Paris Descartes, 45 rue des Saints Pères, 75006 Paris, France. mounira.chelbi-alix@ parisdescartes.fr

transcription) et induisent l'expression de plus d'une centaine de gènes appelés ISG (IFN-stimulated gene), dont les produits sont les médiateurs des différents effets biologiques des I'IFN.

L'IFN de type I se lie à la surface des cellules à des récepteurs spécifiques (IFN alpha and beta receptors) qui sont constitués de deux sous-unités transmembranaires, sous-unités 1 et 2 , codées respectivement par les gènes IFNAR1 et IFNAR2. Cette reconnaissance conduit à l'activation des protéines de la famille des Janus kinases, Jakl et Tyk2 (tyrosine kinase 2), qui activent à leur tour les facteurs de transcription STATl et STAT2. Phosphorylées, STATl et STAT2 forment des hétérodimères (STAT1:STAT2) ou des homodimères (STAT2:STAT2) qui se lient à IRF9 (interferon regulorary factor 9). Les complexes ainsi formés migrent vers le noyau, puis se fixent alors sur une séquence d'ADN spécifique, nommée ISRE (IFN-stimulated response element), et activent la transcription des gènes induits par les IFN de type I.

La reconnaissance de I'IFN de type II par ses récepteurs (IFN gamma receptors), constitués des sous-unités IFNGR1 et IFNGR2, active les kinases Jakl et Jak2 qui phosphorylent STATl, induisant son homodimérisation (STATI:STAT1). Les dimères ainsi formés migrent ensuite vers le noyau, se fixent sur une séquence d'ADN particulière, GAS (gamma activation site), et activent la transcription des gènes induits par l'IFN de type II.

Les IFN de type III, bien que se fixant à des récepteurs spécifiques, activent la même voie de signalisation que l'IFN de type I.

L'IFN induit l'apoptose dans les cellules de différentes lignées cellulaires par un mécanisme impliquant des caspases. Cette apoptose apparaît tardivement après la stimulation par l'IFN car elle nécessite l'augmentation des produits des ISG, dont la protéine PML (promyelocytic leukemia) et la caspase 8, impliquées dans l'apoptose induite par l'IFNo [2]. La diminution de PML, par ARN interférence, bloque ainsi l'apoptose induite par l'IFN $\alpha$ dans les cellules de myélome [3]. 


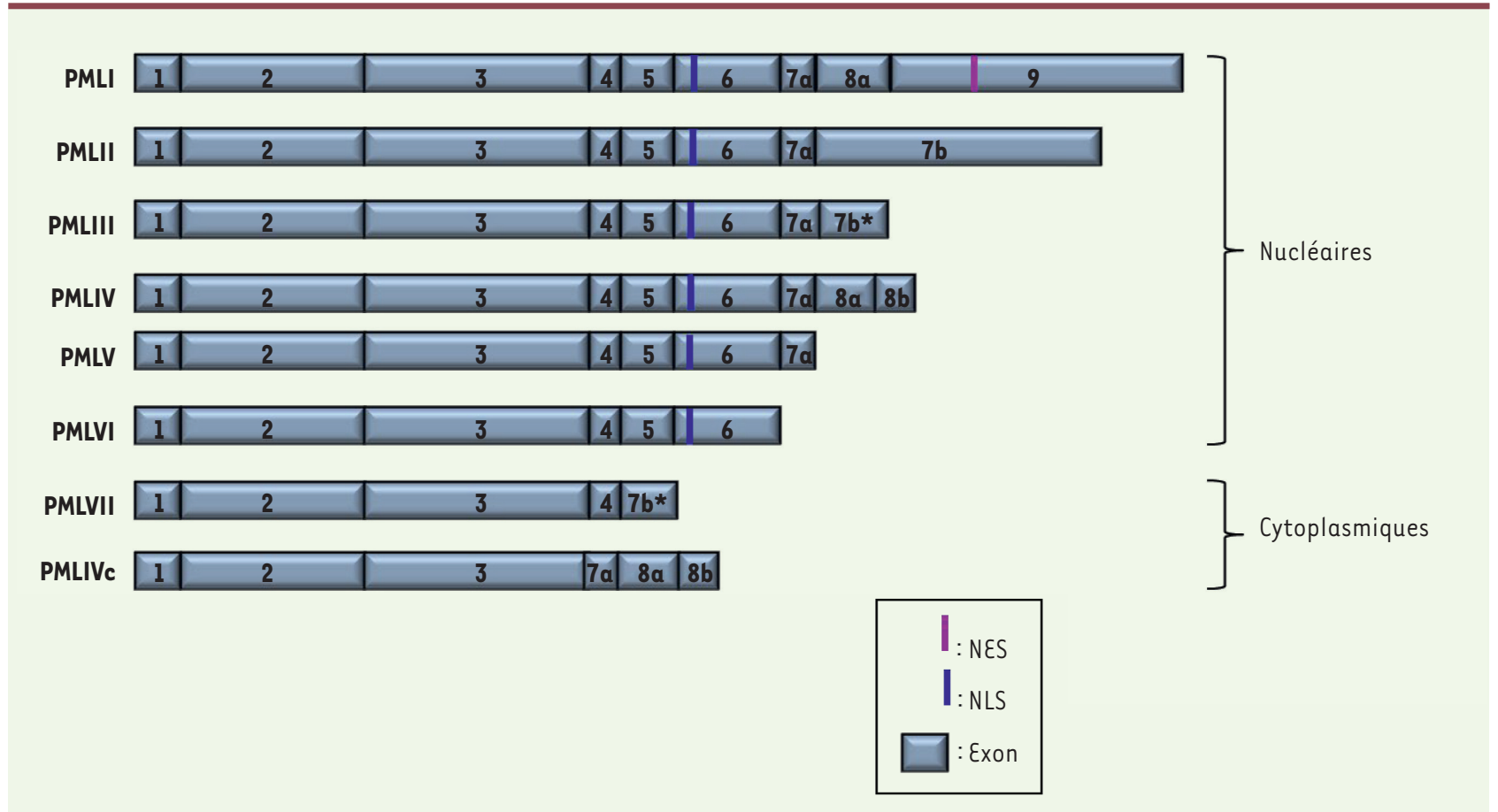

Figure 1. Les isoformes de PML. Le gène PML est constitué de 9 exons (noté de 1 à 9 ), les exons 7 et 8 sont subdivisés en exons $7 a, 7 b, 8 a$ et $8 b$. Les transcrits issus de ce gène génèrent 7 isoformes principales (PMLI à PMLVII) par épissage alternatif des exons 4 à 9 . Les isoformes PMLI à PMLVI, toutes pourvues d'une séquence NLS (nuclear localization sequence), sont nucléaires, alors que PMLVII, dépourvue de ce signal, est strictement cytoplasmique. PMLIVc est cytoplasmique et correspond à PMLIV sans les exons 4, 5 et 6.

\section{Les isoformes de PML et les corps nucléaires PML}

PML, appelée aussi TRIM19 (tripartite motif protein 19), appartient à la famille de protéines RBCC (RING-B box-coiled-coil) ou TRIM (tripartite motif). Comme ces protéines, PML se caractérise par la présence dans sa séquence de quatre motifs: un domaine RING finger, impliqué dans ses interactions avec d'autres protéines; deux boîtes $B$ ( $B$ box); et un domaine coiled-coil, responsable de sa multimérisation. Du fait d'épissages alternatifs entre les exons 4 et 9 à partir d'un gène unique, 7 isoformes principales de PML sont générées, dont 6 sont nucléaires (PMLI à PMLVI), possédant un motif NLS (nuclear localisation signal), et une, dénuée du motif NLS, cytoplasmique (PMLVII) (Figure 1) [4,5]. D’autres isoformes de PML pourraient également être exprimées par épissage alternatif des exons 4,5 et 6 . Dans la nomenclature de PML, des lettres peuvent être ajoutées pour indiquer la perte d'exons : « $a$ » correspond aux isoformes sans l'exon $5, \ll b »$ aux isoformes sans les exons 5 et 6 , et $\langle c »$ aux isoformes sans les exons 4,5 et 6 . Les isoformes $« b$ » et $\langle c »$ sont cytoplasmiques car elles n'ont pas l'exon 6 qui contient le motif NLS $[4,5]$. Ainsi, PMLIVc correspond à PMLIV sans les exons 4,5 et 6 ; elle est donc cytoplasmique.

Les is oformes de PML ont en commun la même région $\mathrm{N}$-terminale (exons 1-3) qui contient le motif RBCC. Elles diffèrent au niveau de leur extrémité C-terminale [5] (Figure 1). Cette variabilité de la région C-terminale de PML explique les interactions privilégiées avec certains partenaires et les fonctions spécifiques que présentent certaines isoformes de PML [5-8]. Par exemple, une étude comparative des 7 isoformes majeures de PML a révélé que seule PMLIV est impliquée dans l'immunité innée, en régulant positivement la phosphorylation d'IRF3 (IFN regulatory factor 3 ) en réponse à une infection virale, ce qui conduit à une plus forte induction d'IFN $[6,9](\rightarrow)$.

$(\rightarrow)$ Voir la Synthèse de M.A. Maroui et al., $m / s n^{\circ} 8-9$, aoûtseptembre 2014, page 765

D'un point de vue mécanistique, PMLIV se lie spécifiquement à Pinl (prolyl isomerase) et la recrute au sein des corps nucléaires PML. Ceci empêche Pinl d'interagir avec la forme phosphorylée d'IRF3, d'entraîner sa dégradation et conduit donc à une augmentation d'IRF3 phosphorylée suite à une infection virale [6].

Dans le noyau, PML est majoritairement sous une forme diffuse dans le nucléoplasme et sous une forme associée à la matrice nucléaire, appelée corps nucléaires PML (CN PML, ou, en anglais, PML NB pour nuclear bodies). Les $\mathrm{CN}$ sont des complexes multiprotéiques organisés par PML. Ils sont constitués de protéines résidentes de façon permanentes, comme PML, Sp100 et Daxx (death domain-associated protein), dont l'expression est augmentée en réponse à l'IFN et d'une multitude de protéines recrutées de façon transitoire en réponse à divers traitements. 
Les éléments de réponses ISRE et GAS sont présents au niveau du promoteur du gène $P M L$. Son expression est donc directement induite par tous les IFN [10], qui sont ainsi à l'origine de l'augmentation de l'expression des isoformes de PML et de l'accroissement en nombre et en taille des CN PML [11]. PML est ensuite modifiée de façon covalente par SUMO (small ubiquitin modifier), une protéine de la famille des ubiquitines. Cette modification post-traductionnelle est essentielle à la fonction des CN PML : PML et CN sont impliqués dans différents processus cellulaires, tels que l'apoptose, la sénescence, la différenciation et la stabilité du génome [12-15] $(\rightarrow)$.

$\rightarrow$ Voir la Nouvelle de N. Martin et al., $m / s n^{\circ} 3$, mars 2102 , page 248

Ils participent également à la réponse à l'IFN et à la défense antivirale $[9,16-18]$.

Dans les cellules traitées par l'IFN, les isoformes de PML sont exprimées principalement dans le nucléoplasme, une petite fraction étant localisée dans la matrice nucléaire où sont associés les CN PML [19-21]. Le transfert de PML du nucléoplasme vers la matrice nucléaire repose sur sa SUMOylation. La SUMOylation de PML est donc importante pour sa localisation. Elle est également importante pour les fonctions des CN $P M L$, telles que le recrutement des partenaires de PML, la dégradation de protéines et la défense antivirale [5-7]. La modification de PML par SUMO et son transfert du nucléoplasme vers la matrice nucléaire sont augmentés par un traitement des cellules par le trioxyde d'arsenic $\left(\mathrm{As}_{2} \mathrm{O}_{3}\right)$, utilisé dans le traitement de la leucémie aiguë promyélocytaire [22], ou par le TGF- $\beta$ (transforming growth factor) [21], ainsi que par l'infection des cellules par le poliovirus ou le virus de l'encéphalomyocardite $[20,23]$.

\section{Le TGF- $\beta$}

Le TGF- $\beta$ (transforming growth factor) contrôle la prolifération et la différenciation cellulaire, et l'induction de l'apoptose dans la plupart des cellules. Il déclenche un signal intracellulaire via l'activation et l'hétérodimérisation des deux récepteurs spécifiques, les $T \beta R$ (transmembrane serine/threonine kinase receptors)-I et II auxquels il se lie. Le complexe formé est ensuite internalisé et est retrouvé dans les endosomes précoces en s'associant au facteur protéique endocytique SARA (SMAD anchor for receptor activation). Ce processus induit la phosphorylation des protéines SMAD2/3 et il est facilité par l'association à SARA. Les protéines SMAD2/3 phosphorylées forment alors avec le facteur de transcription SMAD4 un complexe qui est transloqué vers le noyau, aboutissant à la transcription des gènes cibles du TGF- $\beta$ [24]. À noter que l'altération de la voie de signalisation du TGF- $\beta$ est liée à la physiopathologie de plusieurs cancers [25].

L'activation de la caspase 8 joue un rôle essentiel dans l'apoptose induite en réponse au TGF- $\beta[26,27]$. La SUMOylation de la caspase 8 , au niveau de sa lysine 156, modifie la localisation de la protéine : la forme non SUMOylée ( $p 55$ ) de la caspase 8 est en effet essentiellement présente dans le cytoplasme, alors que la forme SUMOylée (p75) est exclusivement nucléaire. Elle conserve cependant sa capacité d'être activée [28].

\section{Les isoformes de PML et leur implication dans la réponse au TGF- $\beta$}

PML joue un rôle clé dans la réponse au TGF- $\beta$. Les cellules primaires isolées de souris dont le gène $P M L$ a été invalidé sont en effet résistantes à l'arrêt de la croissance et à l'apoptose induites par le TGF- $\beta$ [29] (Figure 2). Cette observation a ainsi permis de révéler le rôle de PML dans la réponse au TGF- $\beta$, dans le cytoplasme [30] et plus récemment dans le noyau [21].

\section{PML cytoplasmique et la voie de signalisation du TGF- $\beta$}

Les cellules isolées de souris dans lesquelles le gène $P M L$ a été invalidé ( $P \mathrm{ML}^{-/-}$) deviennent insensibles à l'apoptose en réponse au TGF- $\beta$ [29]. Cette absence de réponse a pour origine une altération du signal de transduction induit par le TGF- $\beta$ et une diminution de l'induction des gènes cibles [30]. En effet, dans les cellules $\mathrm{PML}^{-/-}$, la phosphorylation des protéines SMAD2/3 en réponse au TGF- $\beta$ est plus faible que dans les cellules parentales qui expriment $\mathrm{PML}$ $\left(\mathrm{PML}^{+/+}\right)$. De façon remarquable, l'introduction dans les cellules $\mathrm{PML}^{-/-}$de la forme cytoplasmique de $\mathrm{PML}$ (PMLc ou PMLIVc), mais pas de sa forme nucléaire (PMLIV), restaure l'effet stimulant du TGF- $\beta$ sur la phosphorylation de SMAD2/3 [30]. De même, la surexpression de PMLc dans des cellules $\mathrm{PML}^{+/+}$régule positivement la signalisation induite par le TGF- $\beta$. $\varepsilon n$ effet, en réponse au TGF- $\beta$, PMLc est localisée au niveau des endosomes précoces où elle s'associe au complexe T $\beta R-I / I I / S A R A / S M A D 2 / 3$ [30]. PMLc contrôle ainsi l'accumulation de SARA et du récepteur du TGF- $\beta$, et facilite l'interaction des protéines SMAD2/3 avec SARA, ce qui augmente leur phosphorylation [30] (Figure 2).

La régulation de l'activité biologique du TGF- $\beta$ est essentielle pour le maintien de l'homéostasie de la cellule. Plusieurs régulateurs ciblant différents acteurs de la voie du TGF- $\beta$ ont été décrits et leurs mécanismes explorés. Ainsi, l'association de SMAD7 avec le récepteur T $\beta R$ I empêche l'activation de SMAD2 bloquant la réponse de la cellule au TGF- $\beta[31,32]$. La protéine TGIF (TG-interacting factor) régule négativement la voie $\mathrm{du}$ TGF- $\beta$ en interagissant avec PMLc et en la séquestrant dans le noyau, ce qui empêche l'interaction de PMLc avec SARA et inhibe la phosphorylation de SMAD2 ainsi que la propagation du signal induit par le TGF- $\beta$ [33]. L'interaction de TGIF avec PMLc est facilitée en présence de la protéine $c$-jun. Cependant, une autre protéine suppresseur de tumeur PHRFl (PHD and RING finger domains 1), qui présente une activité ubiquitine ligase, 


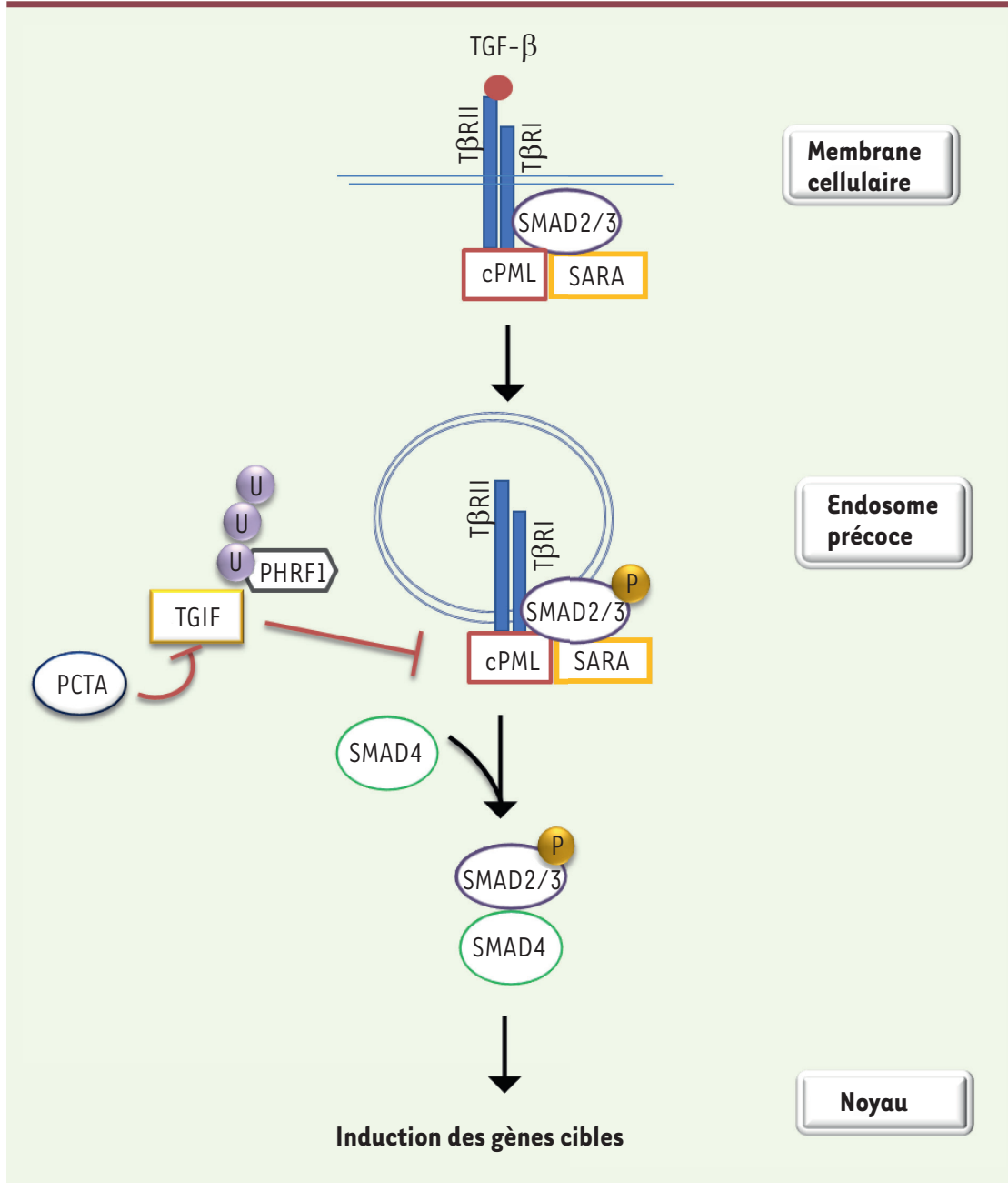

Figure 2. PMLc régule positivement le signal de transduction du TGF- $\beta$. En réponse au TGF$\beta$, PMLc forme un complexe avec SARA (SMAD anchor for receptor activation) et SMAD2/3, ce qui conduit à la localisation des récepteurs du TGF- $\beta$ (T $\beta$ RI/ T $\beta$ RII) au niveau des endosomes précoces, permettant la phosphorylation de SMAD2/3 qui forment avec le facteur de transcription SMAD4 un complexe. Ce complexe est transloqué vers le noyau, aboutissant à la transcription des gènes cibles du TGF- $\beta$. La protéine TGIF (TG-interacting factor) contrôle négativement l'action de PMLc : elle séquestre PMLc dans le noyau empêchant son interaction avec SARA et la phosphorylation de SMAD2/3. PHRFI (PHD and RING finger domains 1 ) induit l'ubiquitination et la dégradation de TGIF et assure la redistribution de PMLc dans le cytoplasme. PCTA (PML competitor for TGIF association), un autre régulateur négatif de la voie de signalisation du TGF- $\beta$, s'associe à TGIF et empêche son interaction avec CPML qui s'accumule dans le cytoplasme. U : ubiquitine.

Ce processus aboutit à des temps plus tardifs à la dégradation de PML nucléaire et à la disparition des CN PML [21].

L'effet du TGF- $\beta$ sur PML nucléaire est séquentiel et se résume comme suit (Figure 3) :

régule positivement la voie de signalisation induite par le TGF- $\beta$ en provoquant la dégradation de TGIF et en assurant par conséquent la redistribution de PMLc dans le cytoplasme [34]. PCTA (PML competitor for TGIF association), une protéine antagoniste du TGIF, peut s'associer à TGIF, empêchant son interaction avec PMLc, ce qui conduit à l'accumulation de PMLc dans le cytoplasme [35]. D'autres $\varepsilon 3$ ubiquitine ligases participent à la régulation du TGF- $\beta$. Tiull (TGIF-interacting ubiquitin ligase 1 ) inhibe en effet le signal de transduction du TGF- $\beta$ à deux niveaux : elle dégrade SMAD2 en s'associant à TGIF, et T $\beta R$-I en s'associant à SMAD7 [36]. SMURF (SMAD-specific $\varepsilon 3$ ubiquitin protein ligase)-1 et -2 sont deux $\varepsilon 3$ ubiquitine ligases de la famille NEDD4 (neural precursor cell-expressed developmentally downregulated gene 4), également impliquées dans la régulation négative du TGF- $\beta$ via la dégradation de SMAD2 [37] et de T $\beta R-1[38,39]$.

\section{Corps nucléaires PML et réponse au TGF- $\beta$}

Le rôle des CN PML dans la réponse au TGF- $\beta$ était inconnu il y a encore peu de temps. Dans une étude récente, nous avons montré que le prétraitement des cellules humaines de la lignée de lymphome de Burkitt (BL41) avec l'IFN $\alpha$ augmente l'apoptose et l'activation de caspase 8 en réponse au TGF- $\beta$ sans altérer le niveau de phosphorylation des protéines SMAD2.

- Le TGF- $\beta$ induit le transfert de PML et de caspase 8 vers la matrice nucléaire

Dans le noyau des cellules traitées par l'IFN $\alpha$, les isoformes de PML sont exprimées principalement dans le nucléoplasme, une petite fraction étant dans la matrice nucléaire à laquelle sont associées les CN PML [19-21]. De façon remarquable, l'addition du TGF- $\beta$ durant $15 \mathrm{~h}$ aux cellules prétraitées par l'IFN $\alpha$ induit le transfert de PML du nucléoplasme vers la matrice nucléaire où sont retrouvées les formes SUMOylées de PML [21]. Le TGF- $\beta$ induit la SUMOylation des isoformes nucléaires de PML (PMLIII et PMLIV) ainsi que leur transfert vers la matrice nucléaire. Ce processus est accompagné par l'apparition de la forme SUMOylée (p75) et des formes activées ( $p 45$ et p20) de la caspase 8 dans la matrice nucléaire des cellules qui surexpriment PMLIV mais pas dans celles qui surexpriment PMLIII.

- PML interagit avec la caspase 8 et la recrute au sein des CN PML

L'addition du TGF- $\beta$ pendant $15 \mathrm{~h}$ aux cellules prétraitées par l'IFN $\alpha$ induit l'interaction de PML avec la caspase 8, 


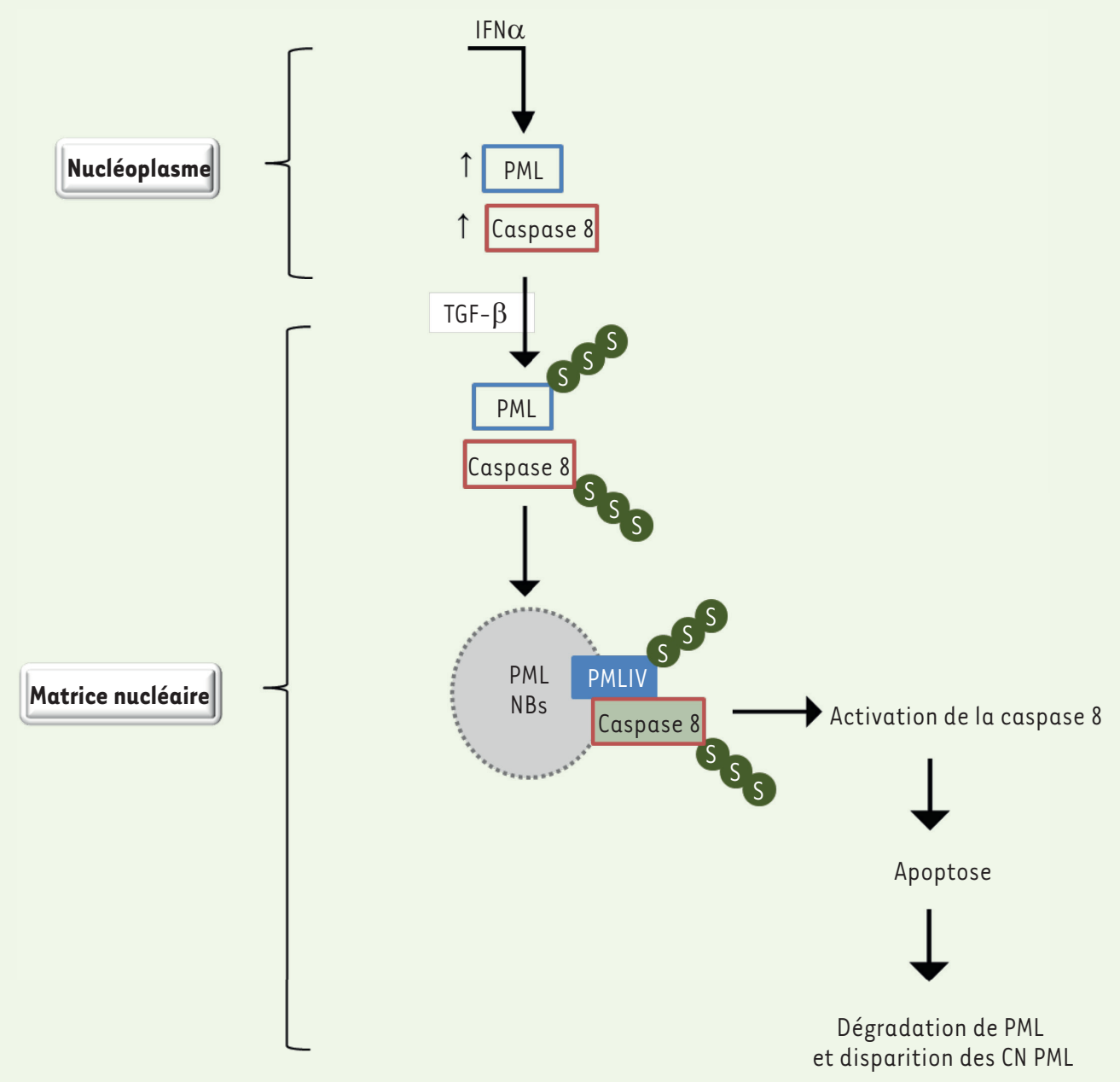

Figure 3. Le prétraitement à l'IFNa augmente l'activation de caspase 8 et l'apoptose en réponse au TGF- $\beta$. 1. L'IFN augmente l'expression de la caspase 8 et des isoformes de PML. 2. L'addition du TGF- $\beta$ induit le transfert de PML et de la caspase 8 vers la matrice nucléaire où sont retrouvées les formes SUMOylés de PML et de la caspase 8. 3. PMLIV recrute la caspase 8 au sein des CN PML. 4. La région C-terminale de PMLIV est requise pour l'interaction avec la caspase 8 et son recrutement dans les CN PML. 5. Ce processus aboutit à l'augmentation de l'activation de la caspase 8 et de l'apoptose en réponse au TGF- $\beta$ et est suivi de la dégradation de PML et de la disparition des CN PML.

et une colocalisation observée par immunofluorescence des deux protéines au sein des CN PML. L'isoforme nucléaire de PML qui interagit avec la caspase 8 a un poids moléculaire correspondant à celle de PMLIII ou PMLIV $(80 \mathrm{kDa})$. Des expériences de co-immunoprécipitation, réalisées sur des cellules surexprimant PMLIII ou PMLIV, ont néanmoins montré que l'interaction entre caspase et PML était spécifique de PMLIV [21]. De plus, le mutant de PMLIV dépourvu de sa région spécifique $C$-terminale codée par l'exon $8 b$, n'interagit plus avec la caspase 8 et n'est plus recruté au sein des CN PML [21]. En réponse au TGF- $\beta$, PMLIV interagit donc avec la caspase 8, la recrute au sein des CN PML et augmente son activation. PMLIV, l'isoforme de PML la plus étudiée, participe à l'immunité innée $[6,9]$. Elle est également impliquée dans l'apoptose et la défense antivirale: par sa région C-terminale, elle séquestre des protéines virales au sein des CN PML bloquant ainsi la réplication du virus $[7,40]$. PMLIV recrute également $p 53$ au sein des CN PML, et induit son activation via les protéines CBP (acetyltransferase CREB-binding protein) et HIPK2 (homeodomain-interacting protein kinase 2), augmentant ainsi l'apoptose induite par plusieurs stimulus $[8,12,41,42]$.

- L'IFN augmente l'activation de caspase 8 et l'apoptose en réponse au TGF- $\beta$

Le prétraitement de cellules BL4l par I'IFN $\alpha$ augmente leur apoptose et l'activation de la caspase 8 induites par le TGF- $\beta$. Le TGF- $\beta$ cible également PML nucléaire en induisant, plus tardivement, sa dégradation par un mécanisme dépendant des caspases [21]. Le traitement des cellules par un inhibiteur spécifique de la caspase 8 empêche ainsi la dégradation de PML, suggérant que la caspase 8 cible spécifiquement PML. La dégradation de PML dans le noyau est suivie de la disparition des CN PML. Un site de clivage pour les caspases est 
présent au niveau de l'acide aminé 552 de la protéine [43], dans ses isoformes nucléaires mais non dans l'isoforme cytoplasmique.

PML est associée à plusieurs voies apoptotiques qui impliquent l'activation d'autres caspases, la caspase 1 et la caspase 3, en réponse à des traitements par des molécules comme Fas (first apoptosis signal), le TNF- $\alpha$ (tumor necrosis factor), les céramides ou I'IFN [12, 29]. Un lien direct entre PML et caspases a ainsi été mis en évidence en observant la localisation de PML et de la caspase 2 au sein des CN PML [44].

\section{Conclusions}

En raison de leur interaction avec différents partenaires, plusieurs fonctions sont attribuées aux isoformes de PML. Celles-ci sont impliquées dans la réponse au TGF- $\beta$, dans le cytoplasme mais également dans le noyau. Dans le cytoplasme, l'isoforme PMLc régule positivement le signal de transduction induit par le TGF- $\beta$ en augmentant la phosphorylation de SMAD2. Dans le noyau, le TGF- $\beta$ induit le transfert de PMLIV et de la caspase 8 vers la matrice nucléaire, leur SUMOylation et leur localisation dans les CN PML. Cette localisation est observée à la fois dans les cellules traitées par I'IFN et dans les cellules exprimant PMLIV. La région C-terminale spécifique de PMLIV est essentielle à l'interaction de la protéine avec la caspase 8 et à son recrutement au sein des CN PML. La colocalisation de la caspase 8 et de PML dans les $C N$ à la suite de la stimulation des cellules par le TGF- $\beta$ suggère une nouvelle voie nucléaire pour l'activation de la caspase 8 ainsi qu'un rôle des CN PML comme régulateurs de l'apoptose en réponse au TGF- $\beta$.

PML cytoplasmique et PML nucléaire agissent donc par des mécanismes différents afin de moduler la réponse des cellules au TGF- $\beta$. $\diamond$

\section{SUMMARY}

PML isoforms and TGF- $\beta$ response

PML/TRIM19 is the organizer of PML nuclear bodies (NB), a multiprotein complex associated to the nuclear matrix, which recruit a large number of proteins involved in various cellular processes. Alternative splicing from a single $P M L$ gene generates 6 nuclear PML isoforms (PMLI to PMLVI) and one cytoplasmic isoform, PMLVII. Murine PML-null primary cells are resistant to TGF- $\beta$-induced apoptosis. Cytoplasmic PML is an essential activator of TGF- $\beta$ signaling by increasing the phosphorylation of transcription factors SMAD2/3 while nuclear PML plays a role in TGF- $\beta$-induced caspase 8 activation and apoptosis. TGF- $\beta$ targets nuclear PML by inducing its conjugation to SUMO. In the nucleus, PML is mainly expressed in the nucleoplasm with a small fraction in the nuclear matrix. In response to TGF$\beta$, PML and caspase 8 shift to the nuclear matrix, where both PML and caspase 8 colocalise within PML NBs. Here, we review the implication of cytoplasmic and nuclear PML isoforms in TGF- $\beta$ response. $\diamond$

\section{LIENS D'INTÉRÊT}

Les auteurs déclarent n'avoir aucun lien d'intérêt concernant les données publiées dans cet article.

\section{RéFÉRENCES}

1. Chelbi-Alix MK, Wietzerbin J. Interferon, a growing cytokine family: 50 years of interferon research. Biochimie $2007 ; 89: 713-8$.

2. Chawla-Sarkar M, Lindner DJ, Liu YF, et al. Apoptosis and interferons: role of interferon-stimulated genes as mediators of apoptosis. Apoptosis 2003; 8 : 237-49.

3. Crowder C, Dahle $\emptyset$, Davis RE, et al. PML mediates IFN-alpha-induced apoptosis in myeloma by regulating TRAIL induction. Blood $2005 ; 105$ : $1280-7$.

4. Jensen K, Shiels $C$, Freemont PS. PML protein isoforms and the RBCC/TRIM motif. Oncogene $2001 ; 20: 7223-33$.

5. Nisole S, Maroui MA, Mascle XH, et al. Differential Roles of PML Isoforms. Front Oncol $2013 ; 3: 125$.

6. El-Asmi F, Maroui MA, Dutrieux J, et al. Implication of PMLIV in Both Intrinsic and Innate Immunity. PLoS Pathog. $2014 ; 10$.

7. Maroui MA, Pampin M, Chelbi-Alix MK. Promyelocytic leukemia isoform IV confers resistance to encephalomyocarditis virus via the sequestration of 3D polymerase in nuclear bodies. J Virol $2011 ; 85$ : 13164-73.

8. Fogal V, Gostissa M, Sandy P, et al. Regulation of $p 53$ activity in nuclear bodies by a specific PML isoform. EMBO J $2000 ; 19$ : 6185-95.

9. Maroui MA, El-Asmi F, Dutrieux J, et al. Implication des corps nucléaires PML dans l'immunité intrinsèque et innée. Med Sci (Paris) 2014 ; $30: 765-71$.

10. Stadler M, Chelbi-Alix MK, Koken MH, et al. Transcriptional induction of the PML growth suppressor gene by interferons is mediated through an ISRE and a GAS element. Oncogene $1995 ; 11: 2565-73$.

11. Chelbi-Alix MK, Pelicano L, Quignon F, et al. Induction of the PML protein by interferons in normal and APL cells. Leukemia $1995 ; 9$ : 2027-33.

12. Bernardi R, Papa A, Pandolfi PP. Regulation of apoptosis by PML and the PML-NBs. Oncogene $2008 ; 27: 6299-312$.

13. Krieghoff-Henning $\varepsilon$, Hofmann TG. Role of nuclear bodies in apoptosis signalling. Biochim Biophys Acta 2008 ; 1783 : 2185-94.

14. Jin G, Wang Y-J, Lin H-K. Emerging Cellular Functions of Cytoplasmic PML. Front Oncol $2013 ; 3: 147$.

15. Martin N, Dejean A, Bischof O. TBX2, un nouvel acteur dans la sénescence cellulaire induite par PML. Med Sci (Paris) $2012 ; 28$ : 248-50.

16. Regad T, Chelbi-Alix MK. Role and fate of PML nuclear bodies in response to interferon and viral infections. Oncogene $2001 ; 20$ : 7274-86.

17. Everett RD, Chelbi-Alix MK. PML and PML nuclear bodies: implications in antiviral defence. Biochimie $2007 ; 89: 819-30$.

18. Geoffroy MC, Chelbi-Alix MK. Role of promyelocytic leukemia protein in host antiviral defense. Interf Cytokine Res $2011 ; 31$ : 145-58.

19. Porta C, Hadj-Slimane R, Nejmeddine M, et al. Interferons alpha and gamma induce $\mathrm{p} 53$-dependent and $\mathrm{p} 53$-independent apoptosis, respectively. Oncogene $2005 ; 24: 605-15$.

20. McHichi B El, Regad T, Maroui MA, et al. SUMOylation promotes PML degradation during encephalomyocarditis virus infection. J Virol 2010;84: 11634-45.

21. El-Asmi F, El-Mchichi B, Maroui MA, et al. TGF- $\beta$ induces PML SUMOylation, degradation and PML nuclear body disruption. Cytokine 2019; $120: 264-72$

22. Zhu J, Koken MH, Quignon F, et al. Arsenic-induced PML targeting onto nuclear bodies: implications for the treatment of acute promyelocytic leukemia. Proc Natl Acad Sci USA 1997 ; 94 : 3978-83.

23. Pampin M, Simonin $Y$, Blondel B, et al. Cross talk between PML and $p 53$ during poliovirus infection: implications for antiviral defense.J Virol 2006 ; $80: 8582-92$

24. Massague J. TGFbeta signalling in context. Nat Rev Mol Cell Biol 2012 ; 13 : 616-30.

25. Siegel PM, Massagué J. Cytostatic and apoptotic actions of TGF- $\beta$ in homeostasis and cancer. Nat Rev Cancer 2003 ; 3 : 807-20.

26. Inman GJ, Allday MJ. Apoptosis induced by TGF-beta $l$ in Burkitt's lymphoma cells is caspase 8 dependent but is death receptor independent. J Immunol $2000 ; 165: 2500-10$

27. Schrantz N, Bourgeade MF, Mouhamad S, et al. p38-mediated regulation of an Fas-associated death domain protein-independent pathway leading to caspase- 8 activation during TGFbeta-induced apoptosis in human Burkitt lymphoma B cells BL41. Mol Biol Cell $2001 ; 12$ : 3139-51.

28. Besnault-Mascard L, Leprince C, Auffredou MT, et al. Caspase- 8 sumoylation is associated with nuclear localization. Oncogene $2005 ; 24: 3268-73$.

29. Wang ZG, Ruggero D, Ronchetti S, et al. PML is essential for multiple apoptotic pathways. Nat Genet $1998 ; 20: 266-72$. 


\section{RÉFÉRENCES}

30. Lin HK, Bergmann S, Pandolfi PP. Cytoplasmic PML function in TGF-beta signalling. Nature 2004 ; $431: 205-11$.

31. Hayashi H, Abdollah S, Qiu Y, et al. The MAD-Related Protein Smad7 Associates with the TGF Receptor and Functions as an Antagonist of TGF $\beta$ Signaling. Cell 1997 ; 89 : 1165-73.

32. Nakao A, Afrakhte M, Morén A, et al. Identification of Smad7, a TGFbeta-inducible antagonist of TGF-beta signalling. Nature $1997 ; 389: 631-5$.

33. Seo SR, Ferrand N, Faresse N, et al. Nuclear retention of the tumor suppressor CPML by the homeodomain protein TGIF restricts TGF-beta signaling. Mol Cell $2006 ; 23: 547-59$.

34. Ettahar A, Ferrigno 0, Zhang M-Z, et al. Identification of PHRFl as a tumor suppressor that promotes the TGF- $\beta$ cytostatic program through selective release of TGIF-driven PML inactivation. Cell Rep $2013 ; 4: 530-41$.

35. Faresse N, Colland F, Ferrand N, et al. Identification of PCTA, a TGIF antagonist that promotes PML function in TGF-beta signalling. EMBO 2008 ; 27 : 1804-15.

36. Seo SR, Lallemand F, Ferrand N, et al. The novel $\varepsilon 3$ ubiquitin ligase Tiull associates with TGIF to target Smad2 for degradation. EMBO J $2004 ; 23: 3780-92$.

37. Lin X, Liang $M$, Feng XH. Smurf2 is a ubiquitin $\varepsilon 3$ ligase mediating proteasome-dependent degradation of Smad2 in transforming growth factor-beta signaling. J Biol Chem $2000 ; 275$ : 36818-22.

38. Ebisawa T, Fukuchi M, Murakami G, et al. Smurfl interacts with transforming growth factor-beta type I receptor through Smad7 and induces receptor degradation. J Biol Chem 2001 ; 276 : 12477-80.
39. Kavsak P, Rasmussen RK, Causing CG, et al. Smad7 binds to Smurf2 to form an $\varepsilon 3$ ubiquitin ligase that targets the TGF beta receptor for degradation. Mol Cell $2000 ; 6: 1365-75$

40. Reichelt $M$, Wang $L$, Sommer $M$, et al. Entrapment of viral capsids in nuclear PML cages is an intrinsic antiviral host defense against varicella-zoster virus. PLoS Pathog $2011 ; 7$ : el001266.

41. Guo A, Salomoni P, Luo J, et al. The function of PML in p53-dependent apoptosis. Nat Cell Biol $2000 ; 2: 730-6$

42. Pearson $M$, Carbone $R$, Sebastiani $C$, et al. PML regulates $p 53$ acetylation and premature senescence induced by oncogenic Ras. Nature $2000 ; 406: 207-10$

43. Zhu J, Lallemand-Breitenbach V, The $\mathrm{H}$ de. Pathways of retinoic acid- or arsenic trioxide-induced PML/RARalpha catabolism, role of oncogene degradation in disease remission. Oncogene 2001; 20 : 7257-65.

44. Tang J, Xie W, Yang X. Association of caspase- 2 with the promyelocytic leukemia protein nuclear bodies. Cancer Biol Ther $2005 ; 4: 645-9$.

\section{www.myobase.org}

Catalogue en ligne disponible gratuitement sur Internet publié par l'AFM-Téléthon.

Retrouvez facilement toutes les références bibliographiques sur les maladies neuromusculaires, les situations de handicap qu'elles génèrent et leurs aspects psychologiques.

Myobase donne un accès libre à $75 \%$ du

fonds documentaire collecté depuis 1990 représentant plus de 40000 références spécifiques du domaine des maladies neuromusculaires.

$>$ articles de la littérature biomédicale et psycho-sociale

\section{$>$ livres, thèses}

$>$ guides d'associations et rapports institutionnels d'agences internationales

\section{$>$ brèves en français,} synthèses des articles médico-scientifiques internationaux les plus pertinents

\section{$>$ publications}

AFM-Téléthon destinées aux professionnels de santé ou aux personnes atteintes de maladie neuromusculaire et à leur entourage

\section{UN OUTIL ERGONOMIQUE, UNE INTERFACE BILINGUE}

- Laissez-vous guider par les tutoriels

- Lancez une recherche et affinez votre sélection grâce aux filtres

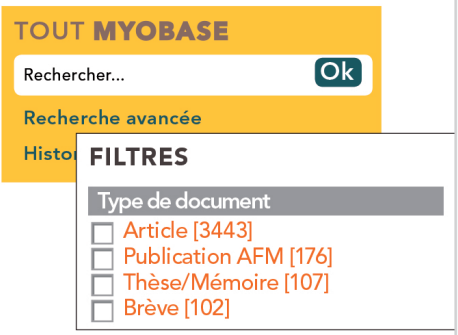

- PUBLICATIONS AFM-Téléthon

$\checkmark$ BRÈVES

- DOCUMENTS DE SYNTHËSE

INSTITUT DES BIOTHÉRAPIES PUBLICATIONS

- Partagez les résultats de votre recherche
TIRÉS À PART

M.K. Chelbi-Alix

\section{UN ACCÈS facile et simple}

Rechercher avec des opérateurs :

- guillemets pour une expression "maladie de pompe"

- + pour signifier ET, et retrouver tous les documents contenant les deux mots "fauteuil +électrique"

- - pour signifier NON et enlever le mot de la recherche :

"autonomie -établissement"

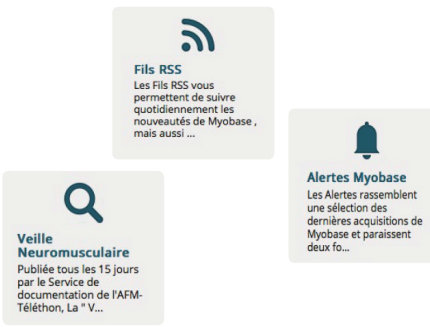

- Cliquez sur I'onglet

thématique qui vous convient (haut de la page d'accueil)

- Créez vos alertes personnalisées en ouvrant un compte personnel

- Téléchargez la Veille

Neuromusculaire

- Abonnez-vous aux flux RSS 